\title{
Heisenberg limit for detecting vacuum birefringence
}

\author{
N. Ahmadiniaz $\odot,{ }^{1}$ T. E. Cowan, ${ }^{1,2}$ R. Sauerbrey, ${ }^{1,3}$ U. Schramm $\odot,{ }^{1,2}$ H.-P. Schlenvoigt $\odot,{ }^{1}$ and R. Schützhold ${ }^{1,4}$ \\ ${ }^{1}$ Helmholtz-Zentrum Dresden-Rossendorf, Bautzner Landstraße 400, 01328 Dresden, Germany \\ ${ }^{2}$ Institut für Kern- und Teilchenphysik, Technische Universität Dresden, 01062 Dresden, Germany \\ ${ }^{3}$ Institut für angewandte Physik, Technische Universität Dresden, 01062 Dresden, Germany \\ ${ }^{4}$ Institut für Theoretische Physik, Technische Universität Dresden, 01062 Dresden, Germany
}

(Received 3 May 2020; accepted 8 June 2020; published 30 June 2020)

\begin{abstract}
Quantum electrodynamics predicts the vacuum to behave as a nonlinear medium, including effects such as birefringence. However, for experimentally available field strengths, this vacuum polarizability is extremely small and thus very hard to measure. In analogy to the Heisenberg limit in quantum metrology, we study the minimum requirements for such a detection in a given strong field (the pump field). Using a laser pulse as the probe field, we find that its energy must exceed a certain threshold depending on the interaction time. However, a detection at that threshold, i.e., the Heisenberg limit, requires highly nonlinear measurement schemes-while for ordinary linear-optics schemes, the required energy (Poisson or shot noise limit) is much larger. Finally, we discuss several currently considered experimental scenarios from this point of view.
\end{abstract}

DOI: 10.1103/PhysRevD.101.116019

\section{INTRODUCTION}

Classical electrodynamics is governed by the Maxwell equations, which are linear in the absence of sources. Thus electromagnetic waves in vacuum obey the superposition principle and do not interact [1]. Quantum electrodynamics, on the other hand, predicts deviations from this behavior: Even the quantum vacuum should be polarizable and thus behave as a nonlinear medium due to the coupling to the fermionic modes; see, e.g., [2-11].

However, since this polarizability is extremely weak for available fields, this fundamental prediction of quantum electrodynamics has not been experimentally verified yet for electromagnetic waves in vacuum (i.e., real photons). Note that an analogous effect has been observed for the interaction of photons with the Coulomb field of atomic nuclei (referred to as Delbrück scattering) [12-16].

Partly motivated by the present-day and near-future experimental facilities aimed at the generation of ultrastrong electromagnetic fields, there have been several proposals and initiatives for verifying this fundamental prediction experimentally; see, e.g., [17-66]. These scenarios include strong magnetic fields [17-26] (see also the recent review [27]), optical or near-optical lasers [28-30], as well as X-ray free electron lasers (XFELs); see, e.g., [31-35]. Various

Published by the American Physical Society under the terms of the Creative Commons Attribution 4.0 International license. Further distribution of this work must maintain attribution to the author(s) and the published article's title, journal citation, and DOI. Funded by SCOAP . potential signatures of the quantum vacuum polarizability have been considered, such as photon polarization changes (birefringence), but also the deflection (scattering) of photons [61] or the phase difference in a Mach-Zehnder-type interferometer setup [30].

In order to compare the different proposals and to sort them into a bigger picture, we address the general question of what are the minimum requirements for detecting the tiny polarizability or nonlinearity of the quantum vacuum. To be more specific, we focus on photon propagation effects and do not consider photon splitting or photon merging or four-wave mixing; cf. [67-84]. Along the lines of quantum metrology, we aim at deriving a fundamental limit for detection, largely independent of the concrete measurement scheme. Apart from the strength of the quantum vacuum polarizability, we find that the available interaction time and the total energy of the probe pulse play an important role. The independence of this limit on the concrete measurement scheme helps us to compare different experimental scenarios and to identify their advantages and drawbacks. Furthermore, preconditions and limitations of this limit point toward ultimate possibilities of increasing sensitivity, even though the required experimental capabilities may be beyond present-day technology.

\section{EULER-HEISENBERG LAGRANGIAN}

Let us start with a brief recapitulation of the basic principles. As motivated above, we focus on slowly varying electromagnetic fields $\mathbf{E}$ and $\mathbf{B}$ well below the Schwinger critical field determined by the electron mass $m$ and the elementary charge $q$ : 


$$
E_{\text {crit }}=\frac{m^{2} c^{3}}{\hbar q} \approx 1.3 \times 10^{18} \frac{\mathrm{V}}{\mathrm{m}}
$$

where the corresponding magnetic field $B_{\text {crit }}=E_{\text {crit }} / c$ is given by $B_{\text {crit }} \approx 4.4 \times 10^{9} \mathrm{~T}$. In this regime [85], the propagation can be described by the lowest-order EulerHeisenberg Lagrangian density $[3,7,8,11,86]$

$\mathcal{L}=\frac{\varepsilon_{0}}{2}\left(\mathbf{E}^{2}-c^{2} \mathbf{B}^{2}\right)+\xi\left[\left(\mathbf{E}^{2}-c^{2} \mathbf{B}^{2}\right)^{2}+7 c^{2}(\mathbf{E} \cdot \mathbf{B})^{2}\right]$,

with the vacuum permittivity $\varepsilon_{0}$ and the prefactor

$$
\xi=\frac{\hbar q^{4}}{360 \pi^{2} m^{4} c^{7}}=\varepsilon_{0} \frac{\alpha_{\mathrm{QED}}}{90 \pi E_{\text {crit }}^{2}}=\frac{2 \alpha_{\mathrm{QED}}^{2}}{45 m^{4}},
$$

where $\alpha_{\mathrm{QED}} \approx 1 / 137$ is the fine-structure constant. From now on, we shall employ natural units with

$$
\hbar=c=\varepsilon_{0}=1,
$$

in order to simplify the expressions.

\section{A. Pump and probe field}

We consider the standard situation where we have a strong (but subcritical) pump field $\mathbf{E}_{0}$ and $\mathbf{B}_{0}$ acting on the vacuum plus a weaker probe pulse $\mathbf{E}_{1}$ and $\mathbf{B}_{1}$ in order to detect the induced polarizability. The pump field is supposed to be a solution of the equations of motion stemming from (2) and we study the propagation of the probe field in this background. Then, inserting the split $\mathbf{E}=\mathbf{E}_{0}+\mathbf{E}_{1}$ and $\mathbf{B}=\mathbf{B}_{0}+\mathbf{B}_{1}$ into (2) and linearizing the equations of motion in $\mathbf{E}_{1}$ and $\mathbf{B}_{1}$, we obtain the well-known effective Lagrangian for the probe field:

$$
\begin{aligned}
\mathcal{L}_{1}= & \frac{1}{2}\left[\mathbf{E}_{1} \cdot(\mathbb{1}+\delta \epsilon) \cdot \mathbf{E}_{1}-\mathbf{B}_{1} \cdot(\mathbb{1}-\delta \mu) \cdot \mathbf{B}_{1}\right] \\
& +\mathbf{E}_{1} \cdot \delta \Psi \cdot \mathbf{B}_{1} .
\end{aligned}
$$

The polarizability of the vacuum is encoded in the change of the dielectric permittivity tensor $\delta \epsilon$ and the magnetic permeability tensor $\delta \mu$ as well as the symmetry-breaking tensor $\delta \Psi$. These quantities depend on the strength of the pump field $\mathbf{E}_{0}$ and $\mathbf{B}_{0}$ (see Appendix $\mathrm{A}$ ) and are suppressed as $\mathcal{O}\left(\alpha_{\mathrm{QED}}\left[\mathbf{E}_{0}^{2}+\mathbf{B}_{0}^{2}\right] / E_{\text {crit }}^{2}\right)$. Since they are very small, we only keep their first order.

Note that we consider the modifications in the propagation of the probe pulse induced by the pump field. Thus, we do not consider other nonlinear QED effects such as photon splitting or merging or four-wave mixing; see, e.g., [67-84], which would correspond to linear or cubic powers of $\mathbf{E}_{1}$ and $\mathbf{B}_{1}$ in (5). In the following, we shall focus on the probe field and consider the tensors $\delta \epsilon, \delta \mu$ and $\delta \Psi$ as externally given. Thus, we shall drop the subscripts for the probe field $\mathbf{E}_{1}$ and $\mathbf{B}_{1}$ from now on.

\section{B. Interaction Hamiltonian}

In terms of the usual vector potential $\mathbf{A}$, the canonically conjugate momentum (which equals the dielectric displacement field $\mathbf{D}$ ) reads

$$
\boldsymbol{\Pi}=\mathbf{D}=(\mathbb{1}+\delta \epsilon) \cdot \mathbf{E}+\delta \Psi \cdot \mathbf{B},
$$

and thus the Hamiltonian density is given by

$$
\begin{aligned}
\mathcal{H}= & \frac{1}{2}[\boldsymbol{\Pi} \cdot(\mathbb{1}-\delta \epsilon) \cdot \boldsymbol{\Pi}+\mathbf{B} \cdot(\mathbb{1}-\delta \mu) \cdot \mathbf{B}] \\
& -\boldsymbol{\Pi} \cdot \delta \Psi \cdot \mathbf{B} .
\end{aligned}
$$

After splitting off the undisturbed vacuum contribution $\mathcal{H}_{0}=\left[\boldsymbol{\Pi}^{2}+\mathbf{B}^{2}\right] / 2$, the remaining part describes the interaction between the probe field and the polarizability $\delta \epsilon, \delta \mu$ and $\delta \Psi$ induced by the pump field

$$
\mathcal{H}_{\text {int }}=-\frac{1}{2} \boldsymbol{\Pi} \cdot \delta \epsilon \cdot \boldsymbol{\Pi}-\frac{1}{2} \mathbf{B} \cdot \delta \mu \cdot \mathbf{B}-\boldsymbol{\Pi} \cdot \delta \Psi \cdot \mathbf{B} .
$$

\section{HEISENBERG LIMIT}

As motivated above, let us now study the question of which minimum requirements the probe pulse has to fulfill in order to detect the vacuum polarizability $\delta \epsilon, \delta \mu$ and $\delta \Psi$. According to the laws of quantum mechanics, this effect is only detectable if the quantum state $|\psi\rangle$ of the probe field after its interaction with the pump field $\hat{U}_{\text {int }}|\psi\rangle$ deviates sufficiently from the quantum state $|\psi\rangle$ without this interaction. As one possible signature, the no-signal fidelity given by (see also [87])

$$
\left\langle\psi\left|\hat{U}_{\mathrm{int}}\right| \psi\right\rangle=\left\langle\psi\left|\mathcal{T} \exp \left\{-i \int d t \hat{H}_{\mathrm{int}}(t)\right\}\right| \psi\right\rangle
$$

should sufficiently deviate from unity ( $\mathcal{T}$ is the timeordering operator). Since the interaction Hamiltonian

$$
\hat{H}_{\text {int }}=\int d^{3} r \hat{\mathcal{H}}_{\text {int }}
$$

is linear in the small tensors $\delta \epsilon, \delta \mu$ and $\delta \Psi$, let us apply first-order perturbation theory:

$$
\left\langle\psi\left|\hat{U}_{\mathrm{int}}\right| \psi\right\rangle=1-i \int d t\left\langle\psi\left|\hat{H}_{\mathrm{int}}(t)\right| \psi\right\rangle+\mathcal{O}\left(\hat{H}_{\mathrm{int}}^{2}\right)
$$

We see that the lowest-order contribution corresponds to a phase shift [87]

$$
\varphi=-\int d t\left\langle\psi\left|\hat{H}_{\mathrm{int}}(t)\right| \psi\right\rangle,
$$

which could be measured by interferometric means, for example; see also [30] and Sec. V below. However, the 
above estimate is just based on the laws of quantum mechanics (and the smallness of $\hat{H}_{\text {int }}$ ) and thus completely independent of the concrete measurement scheme, such as interferometry, change of polarization, or deflection (scattering) of photons. Actually, all those signatures can be related to phase shifts (12) or phase differences; see also Sec. V below. In a Mach-Zehnder-type interferometric setup (see, e.g., [30]), one would measure the phase difference between the two arms (one interacting with the pump field, the other one not). The effect of quantum vacuum birefringence (see, e.g., [31]), i.e., polarization change, is caused by the phase difference between the two polarizations and can also be interpreted as interference between them. Finally, spatial phase differences can change the direction of the probe beam (similar to refraction or diffraction), which may also be exploited for detecting these phenomena (see, e.g., [61] and the recent review [64]). However, as all these phase differences are smaller than the total phase shift (12), i.e., subleading contributions, we focus on this lowest-order contribution in the following.

\section{A. Classical fields}

Let us estimate the maximum possible phase shift (12) for a given probe pulse. First, we treat the probe pulse as a classical field, which should be a good approximation for laser pulses. Inserting Eqs. (10) and (8) into (12), we obtain space-time integrals over the terms $\Pi \cdot \delta \epsilon \cdot \Pi / 2$, $\mathbf{B} \cdot \delta \mu \cdot \mathbf{B} / 2$ and $\boldsymbol{\Pi} \cdot \delta \Psi \cdot \mathbf{B}$. Since the tensor $\delta \epsilon$ is real and symmetric, we may diagonalize it and obtain the bound

$$
\boldsymbol{\Pi} \cdot \delta \epsilon \cdot \boldsymbol{\Pi} \leq \boldsymbol{\Pi}^{2}\|\delta \epsilon\|,
$$

where the norm $\|\delta \epsilon\|$ is the maximum of the absolute values of the eigenvalues of $\delta \epsilon$. In complete analogy, we can bound the term $\mathbf{B} \cdot \delta \mu \cdot \mathbf{B}$ by the same norm $\|\delta \mu\|$ multiplied by $\mathbf{B}^{2}$. Thus, we find

$$
\frac{1}{2}[\boldsymbol{\Pi} \cdot \delta \epsilon \cdot \boldsymbol{\Pi}+\mathbf{B} \cdot \delta \mu \cdot \mathbf{B}] \leq \mathcal{E} \max \{\|\delta \epsilon\|,\|\delta \mu\|\}
$$

at each space-time point, where $\mathcal{E}=\left(\boldsymbol{\Pi}^{2}+\mathbf{B}^{2}\right) / 2$ is the (lowest-order) energy density of the probe pulse.

The remaining term $\boldsymbol{\Pi} \cdot \delta \Psi \cdot \mathbf{B}$ is a bit more complicated because the tensor $\delta \Psi$ is not symmetric in general. Thus, we employ the singular value decomposition

$$
\delta \Psi=\sum_{I} \sigma_{I} \mathbf{u}_{I} \otimes \mathbf{v}_{I}
$$

with the non-negative singular values $\sigma_{I}$ and the two (left and right) orthonormal basis sets $\mathbf{u}_{I}$ and $\mathbf{v}_{I}$. Then, using $\left(\mathbf{u}_{I} \cdot \boldsymbol{\Pi}\right)\left(\mathbf{v}_{I} \cdot \mathbf{B}\right) \leq|\boldsymbol{\Pi}||\mathbf{B}| \leq\left(\boldsymbol{\Pi}^{2}+\mathbf{B}^{2}\right) / 2$, we arrive at

$$
\varphi \leq T \max _{\mathbf{r}}\left\{\sum_{I} \sigma_{I}+\max \{\|\delta \epsilon\|,\|\delta \mu\|\}\right\} \mathfrak{E}
$$

where $T$ denotes the interaction time and $\mathfrak{E}$ the total energy of the probe pulse. The spatial integral can be bounded from above by the maximum over all positions $\mathbf{r}$ since all the involved quantities, such as the energy density $\mathcal{E}$, are non-negative (for classical fields, quantum fields will be discussed in the next section).

Turning the above argument around, we get a minimum energy $\mathfrak{E}$ of the probe pulse required for detecting the vacuum polarizability $\delta \epsilon, \delta \mu$ and $\delta \Psi$ in a given interaction time $T$ since the phase shift $\varphi$ should not be too small in order to achieve a measurable effect. Since the energy $\mathfrak{E}$ scales linearly with the number $N$ of probe photons, we refer to (16) as the (analog of the) Heisenberg limit, a wellknown concept in quantum metrology and interferometry; see also [30].

\section{B. Quantum fields}

In the previous section, we treated the probe pulse as a classical field in order to derive the bound (16). In the following, let us study whether an analogous bound can be established for quantum fields. As a crucial difference, expectation values such as $\left\langle\hat{\boldsymbol{\Pi}}^{2}\right\rangle$ or $\langle\hat{\boldsymbol{\Pi}} \cdot \delta \varepsilon \cdot \hat{\boldsymbol{\Pi}}\rangle$ are divergent and thus require renormalization. As usual, we achieve this by subtracting the vacuum expectation value

$$
\left\langle\hat{\boldsymbol{\Pi}}^{2}\right\rangle_{\text {ren }}=\left\langle\psi\left|\hat{\boldsymbol{\Pi}}^{2}\right| \psi\right\rangle-\left\langle 0\left|\hat{\boldsymbol{\Pi}}^{2}\right| 0\right\rangle .
$$

Of course, this requires appropriate regularization. Here, we use the normal mode decomposition. To this end, we introduce a complete set of orthonormal

$$
\int d^{3} r \mathbf{f}_{I} \cdot \mathbf{f}_{J}=\delta_{I J}
$$

and transversal $\nabla \cdot \mathbf{f}_{I}=0$ basis functions $\mathbf{f}_{I}(\mathbf{r})$ and expand the field operates into this basis set:

$$
\hat{\mathbf{\Pi}}(t, \mathbf{r})=\sum_{I} \hat{p}_{I}(t) \mathbf{f}_{I}(\mathbf{r}) .
$$

Inserting this normal mode decomposition, we find

$$
\begin{aligned}
\int d^{3} r \hat{\boldsymbol{\Pi}} \cdot \delta \varepsilon \cdot \hat{\boldsymbol{\Pi}} & =\sum_{I J} \hat{p}_{I} \hat{p}_{J} \int d^{3} r \mathbf{f}_{I} \cdot \delta \varepsilon \cdot \mathbf{f}_{I} \\
& =\sum_{I J} \hat{p}_{I} \hat{p}_{J} M_{I J}
\end{aligned}
$$

After diagonalizing this real and symmetric matrix $M_{I J}$ via the orthogonal matrix $O_{I J}$, we may introduce a new set of basis functions via $\mathbf{F}_{I}=\sum_{J} O_{I J} \mathbf{f}_{J}$ and expand the field operator in this new set $\hat{\mathbf{\Pi}}(t, \mathbf{r})=\sum_{I} \hat{P}_{I}(t) \mathbf{F}_{I}(\mathbf{r})$ which gives the simplified expression 


$$
\int d^{3} r \hat{\mathbf{\Pi}} \cdot \delta \varepsilon \cdot \hat{\boldsymbol{\Pi}}=\sum_{I} \lambda_{I} \hat{P}_{I}^{2},
$$

where $\lambda_{I}$ are the eigenvalues of the matrix $M_{I J}$. Since the $\hat{P}_{I}^{2}$ are positive operators, we may even derive a (formal) bound on the operator level:

$$
\int d^{3} r \hat{\boldsymbol{\Pi}} \cdot \delta \varepsilon \cdot \hat{\boldsymbol{\Pi}} \leq\|M\| \sum_{I} \hat{P}_{I}^{2}=\|M\| \sum_{I} \hat{p}_{I}^{2},
$$

where $\|M\|=\max _{I}\left|\lambda_{I}\right|$ is the norm of the matrix $M_{I J}$ in analogy to the previous section. It can be estimated by the maximum "expectation value" $\int d^{3} r \mathbf{f} \cdot \delta \varepsilon \cdot \mathbf{f}$ for normalized functions $\mathbf{f}$ and thus agrees with $\max _{\mathbf{r}}\|\delta \varepsilon\|$.

The above operator-valued bound (22) seems to be the proper quantum generalization of the Heisenberg limit (16) since the sums $\frac{1}{2} \sum_{I} \hat{p}_{I}^{2}$ and $\frac{1}{2} \sum_{I} \hat{P}_{I}^{2}$ can be bounded by the total energy of the probe pulse which reads $\frac{1}{2} \sum_{I}\left(\hat{p}_{I}^{2}+\Omega_{I}^{2} \hat{q}_{I}^{2}\right)$ in terms of the eigenmodes $I$ with the eigenfrequencies $\Omega_{I}$. However, this bound is of limited use since the expectation value diverges due to the infinite zeropoint energy (as mentioned above). After subtracting this zero-point energy (17), we cannot deduce the inequality $\left\langle\hat{p}_{I}^{2}\right\rangle_{\text {ren }} \leq\left\langle\hat{p}_{I}^{2}\right\rangle_{\text {ren }}+\Omega_{I}^{2}\left\langle\hat{q}_{I}^{2}\right\rangle_{\text {ren }}$ anymore since the renormalized expectation values $\left\langle\hat{p}_{I}^{2}\right\rangle_{\text {ren }}$ and $\left\langle\hat{q}_{I}^{2}\right\rangle_{\text {ren }}$ can become negative. For example, in a squeezed state $|0\rangle \rightarrow|r\rangle$, we may increase the momentum variance $\left\langle\hat{p}_{I}^{2}\right\rangle \rightarrow \exp \{+r\}\left\langle\hat{p}_{I}^{2}\right\rangle$ while decreasing the position variance $\left\langle\hat{q}_{I}^{2}\right\rangle \rightarrow \exp \{-r\}\left\langle\hat{q}_{I}^{2}\right\rangle$, such that it is below its ground-state value $\left\langle\hat{q}_{I}^{2}\right\rangle<\left\langle 0\left|\hat{q}_{I}^{2}\right| 0\right\rangle$ which means that $\left\langle\hat{q}_{I}^{2}\right\rangle_{\text {ren }}$ becomes negative.

Thus, if we naively replace the classical energy $\mathfrak{E}$ in the Heisenberg limit (16) by the renormalized expectation value $\mathfrak{E}_{\text {ren }}$ for quantum fields, it would be possible to violate this bound by squeezing many modes just a little bit $r \ll 1$ such that their $\left\langle\hat{p}_{I}^{2}\right\rangle_{\text {ren }} \sim r$ increase while the growth of the energy $\mathfrak{E}_{\text {ren }} \sim r^{2}$ is suppressed. One could suspect that this enhancement would be compensated by the other terms such as $\hat{\mathbf{B}} \cdot \delta \mu \cdot \hat{\mathbf{B}}$ which contains $\left\langle\hat{q}_{I}^{2}\right\rangle_{\text {ren }}$ but this is not the case since different modes contribute differently to these terms. Thus, one could squeeze those modes where the first term $\hat{\boldsymbol{\Pi}} \cdot \delta \varepsilon \cdot \hat{\boldsymbol{\Pi}}$ dominates in one way $\left\langle\hat{p}_{I}^{2}\right\rangle \rightarrow$ $\exp \{+r\}\left\langle\hat{p}_{I}^{2}\right\rangle$ and the other modes where the second term $\hat{\mathbf{B}} \cdot \delta \mu \cdot \hat{\mathbf{B}}$ dominates in the opposite way $\left\langle\hat{p}_{I}^{2}\right\rangle \rightarrow$ $\exp \{-r\}\left\langle\hat{p}_{I}^{2}\right\rangle$. For simplicity, we have omitted the third term $\propto \delta \Psi$ since it has yet another mode structure; see also Sec. VA.

In summary, the divergent zero-point energy invalidates a bound like (16) for quantum electrodynamics. To obtain a generalized bound, one would have to limit the number of involved modes $I$ as well as their eigenfrequencies $\Omega_{I}$, which is difficult [88].

\section{COMPARISON TO POISSON LIMIT}

After having derived the Heisenberg limit (16) as our central result, together with its requirements and limitations, we may apply the well-known concepts of quantum metrology and discuss its relation to the Poisson (shotnoise) limit. At first, one might object that a global phase $\varphi$ cannot be measured. While this is correct in principle, this objection could be circumvented by considering a scenario involving a quantum superposition of two paths of the probe pulse, one interacting with the pump field and the other one not. This state corresponds to a many-body entangled NOON state (see, e.g., [89-91]):

$$
|\psi\rangle_{\mathrm{NOON}}=\frac{|N, 0\rangle+|0, N\rangle}{\sqrt{2}},
$$

where either all $N$ probe photons take the one path $|N, 0\rangle$ or all $N$ probe photons take the other path $|0, N\rangle$. Note that this is a highly nonclassical state, in analogy to the GreenbergerHorn-Zeilinger (GHZ) state $[92,93]$. After interaction with the pump field (in one path only), this state evolves into $\left(|N, 0\rangle+e^{i \varphi}|0, N\rangle\right) / \sqrt{2}$ which becomes orthogonal to the initial state (23) for $\varphi=\pi$. Note, however, that both preparing the initial state (23) as well as measuring the final state $\left(|N, 0\rangle+e^{i \varphi}|0, N\rangle\right) / \sqrt{2}$ would require effectively $N$-photon interactions, i.e., a highly nonlinear optics scheme.

In a typical linear-optics setup the (coherent) state of a laser is described by the factorizing state

$$
|\psi\rangle_{\text {laser }}=\bigotimes_{\ell=1}^{N} \frac{|1,0\rangle_{\ell}+|0,1\rangle_{\ell}}{\sqrt{2}},
$$

where each photon $\ell$ individually either takes the one path $|1,0\rangle$ or the other path $|0,1\rangle$. In this case, one would obtain a Poisson distribution of the photon numbers in the output channel and thus the accuracy scales with $1 / \sqrt{N}$ instead of $1 / N$, which is the well-known classical Poisson (shotnoise) limit.

Let us illustrate this distinction in terms of the scaling of the phase with photon number $N$. According to the Heisenberg limit (16), we find

$$
\Delta \varphi_{N}=N \Delta \varphi_{1},
$$

where $\Delta \varphi_{1}$ is the phase shift experienced by a single photon with frequency $\omega$ :

$$
\Delta \varphi_{1}=\omega T \max _{\mathbf{r}}\left\{\sum_{I} \sigma_{I}+\max \{\|\delta \epsilon\|,\|\delta \mu\|\}\right\} .
$$

Since $\Delta \varphi_{N}$ must be of order unity to obtain a measurable detection probability, we get the well-known Heisenberg scaling $\Delta \varphi_{1} \sim 1 / N$. 
In contrast, the Poisson distribution of the photon numbers in the classical (i.e., coherent) state (24) results in a relative accuracy of $1 / \sqrt{N}$ which yields the wellknown Poisson limit $\Delta \varphi_{1} \sim 1 / \sqrt{N}$; see, e.g., [94].

\section{EXPERIMENTAL SCENARIOS}

Now we are in the position to apply our findings to several experimental scenarios known from the literature. Since the Heisenberg limit (16) is independent of the actual measurement scheme, we may apply it directly to the various scenarios in order to compare them and to understand their advantages and drawbacks.

\section{A. Static magnetic pump field}

There are several running or planned experiments where the pump field is an approximately constant magnetic field of a few Tesla; see, e.g., [17-27]. For a purely magnetic field, the symmetry-breaking term $\delta \Psi$ vanishes (see Appendix A). The maximum eigenvalues of the remaining terms $\delta \varepsilon$ and $\delta \mu$ are given by $10 \xi B_{0}^{2}$ and $12 \xi B_{0}^{2}$, respectively, which are then of order $10^{-22}$. Thus, the accuracy requirements are roughly comparable to those for the detection of gravitational waves at LIGO [95]. Indeed, there have been studies for the requirements and possibilities to investigate vacuum birefringence at gravitational wave detectors; see, e.g., [96,97].

As in LIGO, the signal can be amplified by having the probe photons bounce back and forth many times (in a cavity, for example), which facilitates a large integration time $T$. (The noise characterization for cavity-enhanced polarimetry experiments and related issues have been discussed in [98], for example.) Assuming an optimized cavity finesse of order $10^{6}$ and length scales of order meter, we get an integration time of order $\omega T=\mathcal{O}\left(10^{12}\right)$ periods for optical or near-optical photons. Again in analogy to LIGO, the remaining orders of magnitude should be compensated by a sufficiently large number of probe photons. Using the Heisenberg limit (16), we would get $N=\mathcal{O}\left(10^{10}\right)$ which is a comparably low number. However, as explained above, this detection scheme would require an effective $N$-photon interaction involving this number of photons, which is currently out of reach.

With laser fields and linear-optics schemes, we can only reach the Poisson limit, which gives $N=\mathcal{O}\left(10^{20}\right)$ corresponding to a probe pulse energy $\mathfrak{E}$ in the Joule regime. This shows that such an experiment is not impossible with present-day technology but still quite challenging.

Note that the actual limit is even a bit larger because these experiments typically do not measure the polarizabilities $\delta \varepsilon$ and $\delta \mu$ directly, but only the induced rotation of polarization-which measured their difference in the different directions. Otherwise, the rotation of polarization is very similar to an interferometric setup, where the two arms correspond to the two polarizations.

\section{B. Optical pump and XFEL probe}

Another popular scheme envisions a strongly focused ultrastrong laser pulse (again in the optical or near-optical regime) where intensities of order $10^{22} \mathrm{~W} / \mathrm{cm}^{2}$ or more should be reachable with present-day or near-future technology; see, e.g., [99-101]. This corresponds to electric fields above $10^{14} \mathrm{~V} / \mathrm{m}$ which generate polarizabilities $\delta \epsilon$, $\delta \mu$ and $\delta \Psi$ of order $10^{-11}$. This illustrates a major advantage in comparison to the static setup in Sec. VA, as the pump field is much stronger in a laser focus. As a drawback, the interaction time $T$ is limited to the pump pulse length of a few (say ten) optical cycles.

However, for a probe pulse generated by an XFEL with photon energies in the $10 \mathrm{keV}$ range, this corresponds to $\mathcal{O}\left(10^{5}\right)$ XFEL cycles; see, e.g., [32]. The Heisenberg limit (16) then gives $N=\mathcal{O}\left(10^{6}\right)$ photons, i.e., an energy of $\mathcal{O}\left(10^{10} \mathrm{eV}\right)$ or $\mathcal{O}\left(10^{-9} \mathrm{~J}\right)$. Again, as an $\mathrm{N}$-photon interaction with these numbers seems unrealistic, the Poisson limit yields $N=\mathcal{O}\left(10^{12}\right)$ photons, corresponding to an energy of $\mathcal{O}\left(10^{16} \mathrm{eV}\right)$ or $\mathcal{O}\left(10^{-3} \mathrm{~J}\right)$. As before, this shows that the detection is challenging but not completely out of reach.

In analogy to Sec. VA, the envisioned scheme is based on the rotation of the polarization which offers experimental advantages in comparison to an interferometric setup with $\mathrm{x}$ rays but decreases the signal a bit. Note that, with $N=\mathcal{O}\left(10^{11}\right)$ photons in an initially polarized probe beam (see also [102-106]), the signal may consist of a single photon with flipped polarization after several runs. This necessitates a careful study of potentially competing effects in order to distinguish the signal from the background.

\section{Optical pump and optical probe}

In contrast to the scenario described above, it has also been discussed to employ an optical (or near-optical) probe pulse; cf. [30]. Using the same parameters for the pump pulse, the Heisenberg limit (16) would yield the same energy $\mathfrak{E}$ as in Sec. V B. However, the probe pulse would now contain $N=\mathcal{O}\left(10^{10}\right)$ photons because the interaction time corresponds to a few optical cycles only. The Poisson limit then yields $N=\mathcal{O}\left(10^{20}\right)$ corresponding to an energy of $\mathcal{O}\left(10^{20} \mathrm{eV}\right)$ or $\mathcal{O}(10 \mathrm{~J})$. The fact that this is of the same order as the pump pulse itself shows the challenges of this detection scheme.

On the other hand, for this all-optical scheme, it is not necessary to have the optical laser close to an XFEL. Thus, it might be possible to reach even higher intensities in the $10^{23} \mathrm{~W} / \mathrm{cm}^{2}$ regime, which reduces the requirements on the probe pulse to $N=\mathcal{O}\left(10^{18}\right)$ photons, i.e., an energy of $\mathcal{O}\left(10^{18} \mathrm{eV}\right)$ or $\mathcal{O}\left(10^{-1} \mathrm{~J}\right)$. As one possibility, one could use a dual-beam facility (see, e.g., [107]) or spit off a small part of the pump pulse before focusing and use it as probe pulse. This could help ensuring the necessary temporal 
overlap between pump and probe pulse, which can pose a challenge for the scheme described in Sec. V B. Still, performing interference experiments with such $\mathcal{O}\left(10^{-1} \mathrm{~J}\right)$ pulses containing $N=\mathcal{O}\left(10^{18}\right)$ photons is highly nontrivial. Again in analogy to LIGO, it might be advantageous to operate the interferometer not exactly at the dark spot but close to it-corresponding to a small phase mismatch $\varphi_{0}$ between the two arms. For example, using a phase mismatch of $\varphi_{0}=10^{-3}$ (corresponding to a precision of placing the mirrors in the nanometer regime), the output at the dark port would contain $\mathcal{O}\left(10^{12}\right)$ photons. A singlephoton phase shift of $\Delta \varphi_{1}=\mathcal{O}\left(10^{-9}\right)$ would then generate a signal of $\mathcal{O}\left(10^{6}\right)$ photons difference on top of the background of $\mathcal{O}\left(10^{12}\right)$ photons. Measuring such a large photon number with a relative accuracy of $\mathcal{O}\left(10^{-6}\right)$ is challenging and probably requires an advanced detector with many megapixels.

On the other hand, this setup could also offer an advantage as the signal would now contain many $\mathcal{O}\left(10^{6}\right)$ coherent photons, which could help distinguishing it from the background, especially from incoherent noise.

\section{Angular dependence}

Especially for the latter all-optical scenario, it is probably unrealistic to assume a head-on collision between pump and probe pulse. Thus, let us estimate the angular dependence of the phase shift; see also [60]. For simplicity, we model pump and probe pulse as plane waves (as a first step). Then, their relative directions can be described in terms of the three Euler angles $\psi, \theta$, and $\phi$. Without loss of generality, the pump field is supposed to propagate in $z$ direction with $\mathbf{E}_{0}$ and $\mathbf{B}_{0}$ pointing in $x$ and $y$ directions, respectively. After starting with the same orientation, the probe field directions are obtained by three rotations: first, a rotation around the $z$ axis with the angle $\phi$, second, a rotation around the new $x$ axis with the angle $\theta$, followed by a third rotation around the new $z$ axis with the angle $\psi$.

Then, the interaction Hamiltonian reads

$$
\mathcal{H}_{\text {int }}=-2 \xi \mathbf{E}_{0}^{2} \mathbf{E}_{1}^{2}(11-3 \sin [2 \psi-2 \phi]) \sin ^{4} \frac{\theta}{2} .
$$

For the copropagating case $\theta=0$, it vanishes identically (as is well known). In this case, the angles $\psi$ and $\phi$ just rotate the polarization and one can transform into a comoving Lorentz frame where all fields $\mathbf{E}_{0}$ and $\mathbf{B}_{0}$ as well as $\mathbf{E}_{1}$ and $\mathbf{B}_{1}$ become arbitrarily small. The maximum is obtained in the counterpropagating case $\theta=\pi$ where such a Lorentz boost diminishing all fields is not possible. In this limiting case, the angles $\psi$ and $\phi$ again just rotate the polarization and the maximum signal is obtained by $\psi-\phi=-\pi / 4$.

Apart from these well-known limiting cases, we see that this condition $\psi-\phi=-\pi / 4$ does also give the maximum signal for arbitrary given $\theta$, which may be unavoidable due to experimental constraints. For small deviations
$\Delta \theta=\theta-\pi$ from the optimal counterpropagating case $\theta=\pi$, we find

$$
\mathcal{H}_{\text {int }}=-28 \xi \mathbf{E}_{0}^{2} \mathbf{E}_{1}^{2}\left(1-\frac{[\Delta \theta]^{2}}{2}+\mathcal{O}\left([\Delta \theta]^{4}\right)\right) .
$$

\section{CONCLUSIONS}

We study the general requirements for detecting the impact of the weak vacuum polarizability (5) predicted by quantum electrodynamics on the propagation of photons (i.e., the probe pulse). We find that the lowest-order effect is a phase shift (12) which could, at least in principle, be detected by interferometric means. Approximating the probe pulse by a classical field, we obtain an upper bound (16) for the phase shift depending on the interaction time $T$ and the total energy $\mathfrak{E}$ of the probe pulse. Since this phase shift must be of order unity for a measurable detection probability, this inequality does also give the Heisenberg limit (note that $\mathfrak{E} T$ equals the number of photons $N$ times the number of periods $\omega T$ ). However, such a measurement would require a highly nonlinear optics scheme, which is out of reach for realistic parameters. For a linear-optics scheme, we recover the well-known Poisson (shot-noise) limit.

Going beyond the classical field approximation, the failure of proving a bound as (16) for quantum fields hints at the interesting (theoretical) possibility to reach an even higher accuracy by exploiting the zero-point fluctuations. One option could be to squeeze many field modes a little bit such that their quadratures are modified (in order to increase the sensitivity) while the total energy expectation value does not change significantly [108]. For optical frequencies, such a squeezing could be achieved in nonlinear crystals in analogy to parametric down-conversion, while for XFEL frequencies, a corresponding undulator setup could serve the same goal; see also [109]. However, apart from preparing this squeezed state initially, reading out the final state poses grand experimental challenges.

Altogether, we obtain three regimes: the linear-optics regime corresponding to the Poisson (shot-noise) limit, the Heisenberg limit (16) for (locally) classical fields, and a regime beyond that limit for quantum fields.

As a demonstration, we apply these limits to three experimental scenarios, which offer different advantages (e.g., control of polarization for XFEL fields) and disadvantages. For all cases, we find that the detection of the vacuum polarizability is quite challenging but not completely out of reach. Note that, apart from the verification aspect, the vacuum polarizability could also provide a clean way to measure the peak intensity of the laser, which is a highly nontrivial task.

\section{ACKNOWLEDGMENTS}

N. A. gratefully acknowledges helpful discussions with Felix Karbstein and Christian Kohlfürst. R. S. acknowledges 
support from Deutsche Forschungsgemeinschaft (DFG, German Research Foundation, Grant No. 278162697, SFB 1242).

\section{APPENDIX A: VACUUM PERMITTIVITY AND PERMEABILITY TENSORS}

After inserting the split $\mathbf{E}=\mathbf{E}_{0}+\mathbf{E}_{1}$ into $\mathbf{B}=\mathbf{B}_{0}+\mathbf{B}_{1}$ into (2) and keeping the quadratic terms only, we find the effective Lagrangian for the probe field:

$$
\begin{aligned}
\mathcal{L}_{1}= & \xi\left\{4\left(\mathbf{E}_{1} \cdot \mathbf{E}_{0}-\mathbf{B}_{1} \cdot \mathbf{B}_{0}\right)^{2}+2\left(\mathbf{E}_{0}^{2}-\mathbf{B}_{0}^{2}\right)\left(\mathbf{E}_{1}^{2}-\mathbf{B}_{1}^{2}\right)\right. \\
& \left.+7\left(\mathbf{E}_{1} \cdot \mathbf{B}_{0}+\mathbf{B}_{1} \cdot \mathbf{E}_{0}\right)^{2}+14\left(\mathbf{E}_{0} \cdot \mathbf{B}_{0}\right)\left(\mathbf{E}_{1} \cdot \mathbf{B}_{1}\right)\right\} .
\end{aligned}
$$

This effective Lagrangian provides information about the vacuum polarization tensors $(\delta \epsilon, \delta \mu$ and $\delta \psi)$ which can be extracted after comparing with (5)

$$
\mathcal{L}_{1}=\frac{1}{2} \mathbf{E}_{1} \cdot \delta \epsilon \cdot \mathbf{E}_{1}+\frac{1}{2} \mathbf{B}_{1} \cdot \delta \mu \cdot \mathbf{B}_{1}+\mathbf{E}_{1} \cdot \delta \Psi \cdot \mathbf{B}_{1} .
$$

Therefore these tensors are given in terms of the electric and magnetic components of the pump pulse:

$$
\delta \epsilon_{i j}=2 \xi\left(2\left(\mathbf{E}_{0}^{2}-\mathbf{B}_{0}^{2}\right) \delta_{i j}+4 E_{0 i} E_{0 j}+7 B_{0 i} B_{0 j}\right),
$$

which is a symmetric tensor, $\delta \mu$ is obtained from $\delta \epsilon$ by interchanging $\mathbf{E}$ and $\mathbf{B}$ :

$$
\delta \mu=(\delta \epsilon)\left\{E_{i} \leftrightarrow B_{i}, \mathbf{E} \leftrightarrow \mathbf{B}\right\},
$$

and finally the symmetry-breaking tensor

$$
\delta \Psi_{i j}=2 \xi\left(7 \mathbf{E}_{0} \cdot \mathbf{B}_{0} \delta_{i j}+7 E_{0 j} B_{0 i}-4 E_{0 i} B_{0 j}\right) .
$$

As has been already discussed in Sec. III, we need to compute the eigenvalues of $\delta \epsilon$ and $\delta \mu$ and the singular values of $\delta \psi$ since the latter is in general an asymmetric tensor. It is straightforward to obtain the eigenvalues $\lambda_{1,2,3}$ of the symmetric tensors. For a general pump field, we obtain for $\delta \epsilon$

$$
\begin{aligned}
\lambda_{1,2}= & \xi\left(3 \mathbf{B}_{0}^{2}+8 \mathbf{E}_{0}^{2}\right) \\
& \pm \xi \sqrt{49 \mathbf{B}_{0}^{4}+16 \mathbf{E}_{0}^{4}-56 \mathbf{B}_{0}^{2} \mathbf{E}_{0}^{2}+112\left(\mathbf{E}_{0} \cdot \mathbf{B}_{0}\right)^{2}} \\
\lambda_{3}= & 4 \xi\left(\mathbf{E}_{0}^{2}-\mathbf{B}_{0}^{2}\right) .
\end{aligned}
$$

In the limit of constant crossed fields they simplify to

$$
\begin{aligned}
\left|\lambda_{1}\right| & =14 \xi \mathbf{E}_{0}^{2}, \\
\left|\lambda_{2}\right| & =8 \xi \mathbf{E}_{0}^{2}, \\
\lambda_{3} & =0 .
\end{aligned}
$$

Then the spectral representation of $\delta \epsilon$ is

$$
\delta \epsilon=\sum_{I=1}^{3} \lambda_{I} \mathbf{v}_{I} \otimes \mathbf{v}_{I},
$$

where $\mathbf{v}_{I}$ are the eigenvectors of $\delta \epsilon$ (after a suitable rotation of the coordinate system)

$$
\mathbf{v}_{1}=(1,0,0), \quad \mathbf{v}_{2}=(0,1,0), \quad \mathbf{v}_{3}=(0,0,1),
$$

and for $\delta \mu$ (with its eigenvalues are denoted by $\Lambda_{I}$ ) the eigenvalues are simply obtained from the ones for $\delta \epsilon$ by the following replacements:

$$
\begin{aligned}
& \Lambda_{1}=\lambda_{1}\left(E_{i} \leftrightarrow B_{i}\right), \\
& \Lambda_{2}=\lambda_{2}\left(E_{i} \leftrightarrow B_{i}\right), \\
& \Lambda_{3}=\lambda_{3}\left(E_{i} \leftrightarrow B_{i}\right),
\end{aligned}
$$

In the limit of plane-wave background (or constant crossed fields), the two sets of eigenvalues for $\delta \epsilon$ and $\delta \mu$ become equivalent - so to obtain the upper bound, it suffices to consider the maximum value of one of them. Bounding the terms in the Hamiltonian with $\delta \epsilon$ and $\delta \mu$ is simple since they have real eigenvalues; therefore, one can consider the maximum value of their eigenvalues as discussed in Sec. III A. The most nontrivial term is the one with $\delta \psi$ since it has no symmetry in general; then a direct eigenvalue computation may lead to imaginary values which correspond to nonorthogonal set of eigenvectors. Therefore one can rely on singular value decomposition which are defined based on the following theorem [110].

If $A$ is a real $m \times n$ matrix, then there exist two orthogonal matrices $U=\left[\mathbf{u}_{1}, \ldots, \mathbf{u}_{m}\right] \in \mathbb{R}^{m \times m}$ and $V=$ $\left[\mathbf{v}_{1}, \ldots, \mathbf{v}_{n}\right] \in \mathbb{R}^{n \times n}$ such that

$$
U^{T} A V=\operatorname{diag}\left[\sigma_{1}, \ldots, \sigma_{p}\right] \in \mathbb{R}^{m \times n},
$$

where $p=\min \{m, n\}$ and

$$
\sigma_{1} \geq \sigma_{2} \geq \cdots \geq \sigma_{p} \geq 0 .
$$

In other words the singular values $\sigma_{1}, \ldots, \sigma_{p}$ of a $m \times n$ matrix $A$ are the positive square roots, $\sigma_{I}=\sqrt{\lambda_{I}}>0$, of the nonzero eigenvalues of the associated Gram matrix $K=A^{T} A$. The corresponding eigenvectors of $K$ are known as the singular vectors of $A$ (note that for $m \neq n$ or rectangular matrices there is no eigenvalue in its general definition and that is why one finds the singular values).

This theorem leads to the following singular decomposition for a nonsymmetric matrix $A \in \mathbb{R}^{n \times n}$ :

$$
A=\sum_{I=1}^{n} \sigma_{I} \mathbf{u}_{I} \otimes \mathbf{v}_{I}
$$

and from here

$$
A A^{T}=\sum_{I=1}^{n} \sigma_{I}^{2} \mathbf{u}_{I} \otimes \mathbf{u}_{I},
$$


in which $\sigma_{I}$ are the singular values and the left and right singular vectors $u_{I}$ and $v_{I}$ for $I=1,2, \ldots, n$, respectively. Applying this theorem to $\delta \psi$ we get

$$
\begin{aligned}
\sigma_{1,2}^{2}= & 2 \xi^{2}\left[65 \mathbf{E}_{0}^{2} \mathbf{B}_{0}^{2}+84\left(\mathbf{E}_{0} \cdot \mathbf{B}_{0}\right)^{2}\right] \\
& \pm 6 \xi^{2} \sqrt{121 \mathbf{B}_{0}^{4} \mathbf{E}_{0}^{4}+168 \mathbf{E}_{0}^{2} \mathbf{B}_{0}^{2}\left(\mathbf{E}_{0} \cdot \mathbf{B}_{0}\right)^{2}} \\
\sigma_{3}^{2}= & 196 \xi^{2}\left(\mathbf{E}_{0} \cdot \mathbf{B}_{0}\right)^{2} .
\end{aligned}
$$

For a constant crossed field they give

$$
\begin{aligned}
\left|\sigma_{1}\right| & =14 \xi \mathbf{E}_{0}^{2}, \\
\left|\sigma_{2}\right| & =8 \xi \mathbf{E}_{0}^{2}, \\
\sigma_{3} & =0 .
\end{aligned}
$$

$$
\mathcal{A}=\mathcal{B C D}=\left(\begin{array}{c}
\cos \psi \cos \phi-\cos \theta \sin \phi \sin \psi \\
-\sin \psi \cos \phi-\cos \theta \sin \phi \cos \psi \\
\sin \theta \sin \phi
\end{array}\right.
$$

Therefore we have the following equation:

$$
\mathbf{X}=\mathcal{A} \mathbf{x},
$$

where $\mathbf{x}=(x, y, z)$ and $\mathbf{X}=(X, Y, Z)$. To obtain a general form for the interaction Hamiltonian we need the probe electric and magnetic fields $\left(\mathbf{E}_{\omega}, \mathbf{B}_{\omega}\right)$. For a general probe field we have the following transformation:

$$
\begin{aligned}
& \mathbf{E}_{\omega}^{\mathbf{x}}=\mathcal{A} \mathbf{E}_{\omega}^{\mathbf{X}}, \\
& \mathbf{B}_{\omega}^{\mathbf{x}}=\mathcal{A} \mathbf{B}_{\omega}^{\mathbf{X}} .
\end{aligned}
$$

After having the eigenvalues of $\delta \epsilon$ and $\delta \mu$ as well as the singular values of $\delta \psi$ one can easily compute the phase given in (16).

\section{APPENDIX B: THE ROTATION MATRIX}

Let us consider two different frames for the pump and probe pulses in which the former is fixed to be denoted by $x y z$ and the latter $X Y Z$. We need three Euler angles to rotate $X Y Z$ [111]. The sequences of the rotations are the following: $X Y Z$ rotates by an angle $\phi$ about the $Z$ axis to obtain $\xi \eta \zeta$ with corresponding rotation matrix $\mathcal{D}$. For the second rotation, $\xi \eta \zeta$ is rotated about the $\xi$ axes by an angle $\theta$ to obtain new axes called $\xi^{\prime} \eta^{\prime} \zeta^{\prime}$ with rotation matrix $\mathcal{C}$. Finally in the last step the latter is rotated by an angle $\psi$ about $\zeta^{\prime}$ to obtain $x y z$ with rotation matrix $\mathcal{B}$. The three successive rotations lead to a transformation matrix $\mathcal{A}$ :

$$
\left.\begin{array}{cc}
\cos \psi \sin \phi+\cos \theta \cos \phi \sin \psi & \sin \psi \sin \theta \\
-\sin \psi \sin \phi+\cos \theta \cos \phi \cos \psi & \sin \theta \cos \psi \\
-\sin \theta \cos \phi & \cos \theta
\end{array}\right) .
$$

If we consider a constant crossed background for the probe pulse in which the propagation direction lies again in $Z$ and $\mathbf{E}_{\omega}$ and $\mathbf{B}_{\omega}$ in $X$ and $Y$ directions accordingly, then the interaction Hamiltonian defined in (7) with the polarization tensors obtained in Appendix A one arrives at

$\mathcal{H}_{\text {int }}=-2 \xi \mathbf{E}_{0}^{2} \mathbf{E}_{\omega}^{2}(11-3 \sin (2 \psi-2 \phi)) \sin ^{4}\left(\frac{\theta}{2}\right)$,

which has a maximum at $(\theta=\pi, \psi-\phi=-\pi / 4)$.
[1] J. D. Jackson, Classical Electrodynamics, 3rd ed. (Wiley, New York, 1999).

[2] P. A. M. Dirac, Proc. R. Soc. A 117, 610 (1928).

[3] H. Euler and B. Kockel, Naturwissenschaften 23, 246 (1935).

[4] H. Euler, Ann. Phys. (Berlin) 418, 398 (1936).

[5] R. Serber, Phys. Rev. 48, 49 (1935).

[6] E. A. Uehling, Phys. Rev. 48, 55 (1935).

[7] H. Euler and W. Heisenberg, Z. Phys. 98, 714 (1936).

[8] F. Sauter, Z. Phys. 69, 742 (1931).

[9] V. Weisskopf, Kong. Dans. Vid. Selsk, Math-fys. Medd. XIV, 6 (1936).

[10] J. Schwinger, Phys. Rev. 82, 664 (1951).

[11] R. Karplus and M. Neuman, Phys. Rev. 83, 776 (1951).
[12] M. Delbrück, Z. Phys. 84, 144 (1933).

[13] Sh. Zh. Akhmadaliev et al., Phys. Rev. C 58, 2844 (1998).

[14] A. I. Milstein and M. Schumacher, Phys. Rep. 243, 183 (1994).

[15] J. K. Koga and T. Hayakawa, Phys. Rev. Lett. 118, 204801 (2017).

[16] M. Aaboud et al., Nat. Phys. 13, 852 (2017).

[17] http://wwww.toulouse.lncmi.cnrs.fr.

[18] M. T. Hartman, R. Battesti, and C. Rizzo, IEEE Trans. Instrum. Meas. 68, 2268 (2019).

[19] http://www.pvlas.ts.infn.it.

[20] D. Valle, G. Di Domenico, U. Gastaldi, E. Milotti, R. Pengo, G. Ruoso, and G. Zavattini, Opt. Commun. 283, 4194 (2010). 
[21] F. D. Valle, A. Ejlli, U. Gastaldi, G. Messineo, E. Milotti, R. Pengo, G. Ruoso, and G. Zavattini, Eur. Phys. J. C 76, 24 (2016).

[22] H.-H. Mei, W.-T. Ni, S.-J. Chen, and S.-S. Pan (Q\&A), Mod. Phys. Lett. A 25, 983 (2010).

[23] X. Fan et al., Eur. Phys. J. D 71, 308 (2017).

[24] E. Zavattini et al., Phys. Rev. Lett. 96, 110406 (2006).

[25] E. Zavattini et al., Phys. Rev. D 77, 032006 (2008).

[26] E. Zavattini, U. Gastaldi, R. Pengo, G. Ruoso, F. Della Valle, and E. Milotti, Int. J. Mod. Phys. A 27, 1260017 (2012).

[27] A. Ejlli et al., arXiv:2005.12913.

[28] D. Tommasini and H. Michinel, Phys. Rev. A 82, 011803 (2010).

[29] B. King and Ch. Keitel, New J. Phys. 14, 103002 (2012).

[30] S. Ataman, Phys. Rev. A 97, 063811 (2018).

[31] T. Heinzl, B. Liesfeld, K.-U. Amthor, H. Schwoerer, R. Sauerbrey, and A. Wipf, Opt. Commun. 267, 318 (2006).

[32] H.-P. Schlenvoigt, T. Heinzl, U. Schramm, T. E. Cowan, and R. Sauerbrey, Phys. Scr. 91, 023010 (2016).

[33] T. Inada et al., Phys. Lett. B 732, 356 (2014).

[34] T. Inada et al., Appl. Sci. 7, 671 (2017).

[35] T. Yamaji et al., Phys. Lett. B 763, 454 (2016).

[36] W. Becker and H. Mitter, J. Phys. A 8, 1638 (1975).

[37] E. B. Aleksandrov et al., Zh. Eksp. Teor. Fiz. 62, 1181 (1985).

[38] D. Tommasini, A. Ferrando, H. Michinel, and M. Seco, J. High Energy Phys. 11 (2009) 043.

[39] H. Gies, F. Karbstein, and N. Seegert, New J. Phys. 15, 083002 (2013); 17, 043060 (2015).

[40] H. Gies, F. Karbstein, and R. Shaisultanov, Phys. Rev. D 90, 033007 (2014).

[41] R. Battesti et al., Phys. Rep. 765, 1 (2018).

[42] A. Di Piazza, K. Z. Hatsagortsyan, and C. H. Keitel, Phys. Rev. Lett. 97, 083603 (2006).

[43] A. Di Piazza, C. Müller, K. Z. Hatsagortsyan, and C. H. Keitel, Rev. Mod. Phys. 84, 1177 (2012).

[44] K. Homma et al., Romanian reports in Physics 68, S233 (2016).

[45] S. Ataman, M. Cuciuc, L. D’Alessi, L. Neagu, M. Rosu, K. Seto, O. Tesileanu, Y. Xu, and M. Zeng, AIP Conf. Proc. 1852, 070002 (2017).

[46] Y. Nakamiya and K. Homma, Phys. Rev. D 96, 053002 (2017).

[47] V. Dinu, T. Heinzl, A. Ilderton, M. Marklund, and G. Torgrimsson, Phys. Rev. D 89, 125003 (2014).

[48] V. Dinu, T. Heinzl, A. Ilderton, M. Marklund, and G. Torgrimsson, Phys. Rev. D 90, 045025 (2014).

[49] R. P. Mignani, V. Testa, D. González Caniulef, R. Taverna, R. Turolla, S. Zane, and K. Wu, Mon. Not. R. Astron. Soc. 465, 492 (2017).

[50] L. M. Capparelli, A. Damiano, L. Maiani, and A. D. Polosa, Eur. Phys. J. C 77, 754 (2017).

[51] B. Shen, Z. Bu, J. Xu, T. Xu, L. Ji, R. Li, and Z. Xu, Plasma Phys. Controlled Fusion 60, 044002 (2018).

[52] J. J. Klein and B. P. Nigam, Phys. Rev. 135, B1279 (1964).

[53] J. S. Toll, Ph.D. thesis, Princeton, 1952.

[54] R. Baier and P. Breitenlohner, Acta Phys. Austriaca 25, 212 (1967); Nuovo Cimento 47, 261 (1967).
[55] Z. Bialynicka-Birula and I. Bialynicka-Birula, Phys. Rev. D 2, 2341 (1970).

[56] W. Dittrich and H. Gies, Probing the Quantum Vacuum: Perturbative Effective Action Approach in Quantum Electrodynamics and its Application (Springer, New York, 2000), Vol. 166.

[57] G. L. J. A. Rikken and C. Rizzo, Phys. Rev. A 63, 012107 (2000); 67, 015801 (2003).

[58] P. Shukla, M. Marklund, D. Tskhakaya, and B. Eliasson, Phys. Plasmas 11, 3767 (2004).

[59] F. Karbstein, H. Gies, M. Reuter, and M. Zepf, Phys. Rev. D 92, 071301 (2015).

[60] F. Karbstein and C. Sundqvist, Phys. Rev. D 94, 013004 (2016).

[61] F. Karbstein, Phys. Rev. D 98, 056010 (2018).

[62] S. Bargin, S. Meuren, Ch. Keitel, and A. Di Piazza, Phys. Rev. Lett. 119, 250403 (2017).

[63] A. Ilderton and M. Marklund, J. Plasma Phys. 82, 2 (2016).

[64] F. Karbstein, Particles 3, 39 (2020).

[65] V. I. Denisov, E. E. Dolgaya, and V. A. Sokolov, J. High Energy Phys. 05 (2017) 105.

[66] X. Sarazin, F. Couchot, A. Djannati-Ataï, O. Guilbaud, S. Kazamias, M. Pittman, and M. Urban, Eur. Phys. J. D 70, 13 (2016).

[67] S. L. Adler, Ann. Phys. (N.Y.) 67, 599 (1971).

[68] S. L. Adler and C. Schubert, Phys. Rev. Lett. 77, 1695 (1996).

[69] J. M. Dávila, C. Schubert, and M. A. Trejo, Int. J. Mod. Phys. A 29, 1450174 (2014).

[70] Sh. Zh. Akhmadaliev et al., Phys. Rev. Lett. 89, 061802 (2002).

[71] S. L. Adler, J. N. Bahcall, C. G. Callan, and M. N. Rosenbluth, Phys. Rev. Lett. 25, 1061 (1970).

[72] N. N. Rozanov, Zh. Eksp. Teor. Fiz. 103, 1996 (1993) [Sov. Phys. JETP 76, 991 (1993)].

[73] J. Mckenna and P. M. Platzman, Phys. Rev. 129, 2354 (1963).

[74] A. A. Varfolomeev, Zh. Eksp. Teor. Fiz. 50, 1024 (1966).

[75] F. Moulin and D. Bernard, Opt. Commun. 164, 137 (1999).

[76] A. Di Piazza, K. Z. Hatsagortsyan, and C. H. Keitel, Phys. Rev. D 72, 085005 (2005).

[77] A. Di Piazza, A. I. Milstein, and C. H. Keitel, Phys. Rev. A 76, 032103 (2007).

[78] D. Bernard, F. Moulin, F. Amiranoff, A. Braun, J. P. Chambaret, G. Darpentigny, G. Grillon, S. Ranc, and F. Perrone, Eur. Phys. J. D 10, 141 (2000).

[79] E. Lundström, G. Brodin, J. Lundin, M. Marklund, R. Bingham, J. Collier, J. T. Mendonça, and P. Norreys, Phys. Rev. Lett. 96, 083602 (2006).

[80] J. Lundin, M. Marklund, E. Lundström, G. Brodin, J. Collier, R. Bingham, J. T. Mendonça, and P. Norreys, Phys. Rev. A 74, 043821 (2006).

[81] H. Gies, F. Karbstein, and N. Seegert, Phys. Rev. D 93, 085034 (2016).

[82] H. Gies, F. Karbstein, and C. Kohlfürst, Phys. Rev. D 97, 036022 (2018).

[83] B. King, H. Hu, and B. Shen, Phys. Rev. A 98, 023817 (2018).

[84] B. King and T. Heinzl, High Power Laser Sci. Eng. 4, e5 (2016). 
[85] Since the field strength as well as the gradient of the nuclear Coulomb field becomes very large very close to the nucleus and thus violates these conditions, we do not consider Delbrück scattering here.

[86] G. V. Dunne, Heisenberg-Euler effective Lagrangians: basics and extensions, in From Fields to Strings: Circumnavigating Theoretical Physics (World Scientific, Chicago, 2005), Vol. 3, pp. 445-522, https://doi.org/ 10.1142/9789812775344_0014.

[87] R. Schützhold, Phys. Rev. D 98, 105019 (2018).

[88] Note that this problem arises for relativistic quantum fields. For nonrelativistic quantum fields, such as the Schrödinger field $\hat{\Psi}$ describing the atoms in a Bose-Einstein condensate, it is possible to obtain bounds similar to (16). For example, the interaction $\hat{\mathcal{H}}_{\text {int }}$ of a Bose-Einstein condensate with a gravitational wave (or similar disturbances) can be split into the contribution $\hat{\Psi}^{\dagger} \delta V \hat{\Psi}$ stemming from the effective change $\delta V$ of the trap potential plus the term $\left(\nabla \hat{\Psi}^{\dagger}\right) \cdot \delta g \cdot(\nabla \hat{\Psi}) / 2 m$ induced by the variation $\delta g$ of the spatial metric describing the gravitational wave [87]. Due to the absence of a zero-point energy, we may derive a bound in analogy to (16) in terms of the total number of atoms $N$ and the total kinetic energy $\mathfrak{E}_{\text {kin }}$.

[89] P. Kok, H. Lee, and J. P. Dowling, Phys. Rev. A 65, 052104 (2002).

[90] G. J. Pryde and A. G. White, Phys. Rev. A 68, 052315 (2003).

[91] H. Cable and J. P. Dowling, Phys. Rev. Lett. 99, 163604 (2007).

[92] D. M. Greenberger, M. A. Horne, and A. Zeilinger, Am. J. Phys. 58, 1131 (1990).

[93] D. Bouwmeester, J-W. Pan, M. Daniell, H. Weinfurter, and A. Zeilinger, Phys. Rev. Lett. 82, 1345 (1999).
[94] M. Xiao, L-A. Wu, and H. J. Kimble, Phys. Rev. Lett. 59, 278 (1987).

[95] B. P. Abbott et al. (LIGO Scientific and Virgo Collaborations), Phys. Rev. Lett. 116, 061102 (2016).

[96] B. Döbrich and H. Gies, Europhys. Lett. 87, 21002 (2009).

[97] H. Grote, Phys. Rev. D 91, 022002 (2015).

[98] M. T. Hartman, A. Rivère, R. Battesti, and C. Rizzo, Rev. Sci. Instrum. 88, 123114 (2017).

[99] https://www.xfel.eu/ for the European XFEL.

[100] http://corels.ibs.re.kr/ for CoReLS.

[101] https://eli-laser.eu/ for ELI.

[102] T. Tschentscher et al., Appl. Sci. 7, 592 (2017).

[103] I. Agapova et al., arXiv:1404.1177.

[104] E. A. Schneidmiller and M. V. Yurkov, Technical Report No. DESY-11-152, 2011.

[105] B. Marx et al., Phys. Rev. Lett. 110, 254801 (2013).

[106] H. Bernhardt et al., Appl. Phys. Lett. 109, 121106 (2016).

[107] U. Schramm et al., IOP Conf. Series: J. Physics: Conf. Series 874, 012028 (2017).

[108] We would like to stress that there are different scenarios for the application of squeezing here: As is well known, one can "beat" the Poisson limit $\propto 1 / \sqrt{N}$ and improve sensitivity toward the Heisenberg limit $\propto 1 / N$ by squeezing a single mode (or a few modes) strongly. In contrast, by squeezing many modes a little bit, one could even construct a quantum state which violates the Heisenberg limit (16), at least after the natural identification of the (classical) probe pulse energy with the renormalized expectation value (for quantum fields).

[109] R. Schützhold, G. Schaller, and D. Habs, Phys. Rev. Lett. 100, 091301 (2008).

[110] G. H. Golub and C. F. Van Loan, Matrix Computations (Johns Hopkins University Press, Harvard, 2012), Vol. 3.

[111] H. Goldstein, C. Poole, and J. Safko, Classical Mechanics, , 3rd ed. (Addison-Wesley, Reading, MA, 2001). 